\title{
EL OTRO TEATRO DE EMILIA PARDO BAZÁN
}

\author{
THE OTHER THEATRE OF EMILIA PARDO BAZÁN
}

\author{
Ana M. ${ }^{a}$ FREIRE LÓPEZ \\ Universidad Nacional de Educación a Distancia/SELITEN@T \\ afreire@ flog.uned.es
}

Resumen: La autora estudia en este artículo seis obras dramáticas, resultantes de la adaptación a la escena de novelas y cuentos de Emilia Pardo Bazán, realizadas cuando ya la escritora había abandonado la creación dramática, y con ella sus esperanzas de triunfar en el teatro. De la mayor parte de estas adaptaciones no se conserva el texto, pero sí numerosas noticias, que merecen rescatarse y recordarse, si se quiere conocer en toda su amplitud la proyección de la obra de Emilia Pardo Bazán.

Abstract: The author in this article examines six plays, resulting from the stage adaptation of narrative works of Emilia Pardo Bazán, made when the writer had already given up her dramatic creation and her hopes of succeeding in the theater. In most of these adaptations the text has not been preserved, but we have reports that should be known, because they show the projection of the literary work of Emilia Pardo Bazán.

Palabras clave: Emilia Pardo Bazán. Teatro. Adaptaciones escénicas.

Key words: Emilia Pardo Bazán. Dramatic plays. Stage adaptations. 
La creación literaria de Emilia Pardo Bazán suscita desde hace años un interés creciente, y su teatro, que en vida de la escritora no obtuvo el reconocimiento esperado, se ve hoy desde otra perspectiva ${ }^{1}$. Sin embargo, hasta ahora no se ha prestado atención a ese otro teatro de Emilia Pardo Bazán, el que no nació para la escena, sino que, derivado de su obra narrativa, subió a las tablas en adaptaciones realizadas tanto durante su vida como después de su muerte, y de él me ocuparé en este trabajo.

Hoy sabemos que existieron campañas inducidas por quienes no deseaban que doña Emilia triunfara también en el terreno teatral y se conocen los detalles alrededor de cada uno de sus estrenos. Pasados estos con desigual fortuna, Mariano Miguel de Val, en su artículo «Los novelistas en el teatro» (De Val, 1906) defendió con varios ejemplos que muy raramente quien es novelista sirve para dramaturgo. Doña Emilia le rebatió en un artículo con el mismo título, publicado en el siguiente número de la misma revista (Pardo Bazán, 1906), alegando cantidad de buenos dramas escritos por novelistas. Argumentaba De Val que el público a quien se dirigen uno y otro género no es el mismo, y que es necesario plegarse a sus gustos si se quiere triunfar en la escena.

Descontando la malevolencia de sus enemigos, algo de verdad había en esa afirmación. Francisco Nieva, reconociendo la «asombrosa capacidad dramática de la escritora» (Nieva, 1989: 200), también señaló como posible motivo del fracaso de doña Emilia en la escena el que la novelista no escribió un teatro para su tiempo. El paradigma era entonces el de Benavente y, aunque ella fuera consciente de la existencia de aquella fórmula no escrita y tratara de asimilarla, no llegó a acertar con el secreto. Su teatro no ganó al gran público y, de rechazo, su experiencia dramatúrgica la decepcionó a ella misma: «El teatro me ha dejado un desapacible recuerdo. No necesita la escena de mí, y por suerte, no necesito yo, con estricta necesidad, de la escena» (La Nación, 10-12-1916). Meses antes de pronunciar estas palabras, admitía que quizá la causa de su fracaso estuviera en ella, porque el teatro se escribía entonces de otra manera, con moldes que ya no eran los de antes, y también el público era otra cosa: «Y considero dificilísimo acertarle el gusto» (La Acción, 28-2-1916). Muy a su pesar, Emilia Pardo Bazán fue consciente de que su modo de hacer teatro no coincidía con los gustos del momento: "Amo un teatro realista, fuerte, con médula, con sangre,

1 Hasta el momento se han publicado diversos trabajos sobre la creación dramática de doña Emilia y, recientemente, la primera edición anotada y precedida de un estudio de sus obras dramáticas: Emilia Pardo Bazán, Teatro completo (2010). 
precisamente lo que veo que no quiere aceptar el público de nuestro tiempo» (La Nación, 10-12-1916). Esto era tanto como admitir que, poseyendo unos mimbres espléndidos, le faltaba la habilidad para tejerlos al gusto de los espectadores y de la crítica de entonces.

Seguramente por ello y porque siempre le tentó la escena, accedió a que otros convirtieran en obra dramática lo que ella creó en forma narrativa. Y también porque, como ya había escrito en 1906, en su respuesta a De Val:

Cada día se arraiga más en Francia la costumbre de que las novelas algo estimadas de los lectores sean adaptadas a la escena [...] La persistencia del síntoma indica dos cosas: que el público francés no rechaza a los novelistas en la escena, y que la novela va invadiendo el teatro [...] Para no observar este fenómeno habría que cerrar los ojos.

La potencialidad dramática de la escritura de doña Emilia nunca ha sido negada. La creación de personajes, la plasticidad de las escenas, la viveza de los diálogos, el interés y la dosificación de la intriga, el colorismo en la descripción del marco en que se desarrolla la acción son alicientes que mantienen actuales unas novelas escritas, años arriba o abajo, hace alrededor de un siglo. Todo ello facilitó que cuentos y novelas o pasajes significativos de estas fueran convertidos en textos teatrales y llevados a la escena. Su obra narrativa está plagada de escenas dramatizables, porque teatral y llena de vida fue la percepción que doña Emilia tuvo de ellas, aunque no las escribiera pensando en su representación ${ }^{2}$.

Es posible que se hayan llevado a las tablas más obras narrativas de Emilia Pardo Bazán que las que yo he localizado, que son casi tantas como las que ella escribió para el teatro ${ }^{3}$. De alguna adaptación no tengo más noticia que un brevísimo comentario de la propia escritora. Así, refiriéndose a su cuento Nieto del Cid, afirmó que se había leído y traducido bastante, y que de él «hicieron en Francia un dramita en un acto» (La Ilustración Artística, 12-1-1903). Esta es además la única noticia hallada sobre la adaptación a la escena de una obra

${ }^{2}$ Francisco Nieva, refiriéndose al drama Verdad, destacó su «poderosa imaginación específicamente teatral en su forma de conducir el tema», que pone de manifiesto la «asombrosa capacidad dramática de la escritora» (Nieva 1989: 200).

${ }^{3}$ Estas fueron El vestido de boda, La suerte, Verdad, Cuesta abajo, El becerro de metal, Juventud y Las raíces; además de otras que se conservan incompletas, y de una hoy perdida, de las cuales solo cinco llegaron a los escenarios, cuatro en vida de la escritora y El becerro de metal después de su muerte. 
narrativa suya en otro idioma, lo que no significa que no haya habido más. Las obras de las que ahora me ocuparé se adaptaron al teatro en España y en castellano. De la mayor parte de ellas no se conserva el texto dramático, pero sí numerosas noticias, que merecen rescatarse y recordarse, si se quiere conocer en toda su amplitud la proyección de la obra creativa de Emilia Pardo Bazán.

La más antigua es La nota de color, comedia en un acto y en prosa, que se estrenó en el Teatro Eslava de Valencia el 17 de enero de 1911. Era la adaptación a la escena de un capítulo de La Quimera, realizada por el escritor valenciano Antonio Sotillo ${ }^{4}$. El texto dramático no llegó a editarse y, por tanto, no sabríamos de qué capítulo de la novela se trataba si no contáramos con una valiosa ayuda, en este caso gráfica, de la revista Nuevo Mundo (26-1-1911). Es una fotografía que muestra una escena fácilmente localizable en el capítulo V, con verdaderas posibilidades de ser dramatizada. Quienes hayan leído la novela recordarán la visita que Silvio Lago hace a Espina Porcel, cuando es invitado por ella a una velada de sociedad en la que exhibiría un retrato que le habían hecho recientemente. Silvio, sin dudar que se trataría del que él había pintado, se sorprende cuando, al llegar a casa de Espina, la condesa de los Pirineos, que acaba de contemplarlo, no manifiesta sobre el cuadro una opinión muy favorable y, aturdido, se entera de que es el retrato pintado por Marbley. La condesa, al advertir el premeditado desaire de la Porcel, decide darle un escarmiento y le pide que les muestre, a ella y a los demás invitados, el retrato que pintó Silvio, arrinconado en la zona de servicio. A partir de ese momento, la condesa toma a Silvio Lago bajo su protección, encargándole que le haga un retrato y asegurándole que aconsejará lo mismo a sus amigas, mientras con un lapicerito de oro tacha a Espina Porcel del carnet donde tiene anotados los nombres de todas ellas. Como otros momentos precedentes, el final del episodio tiene, en el relato de Pardo Bazán, no solo gran plasticidad sino lo que bien podría tomarse como indicaciones precisas para el actor que interpretase el papel del pintor:

Silvio se quedó de pie en la acera, palpitando de un gozo y de una esperanza que le movían a alzar los ojos hacia el firmamento, alto, estrellado y frío, con este gesto que hacemos involuntariamente para referir nuestras

\footnotetext{
4 Antonio Sotillo fue autor de numerosas comedias, muchas de ellas en colaboración, y de adaptaciones de obras extranjeras. La partitura de su zarzuela La dama roja es de Ruperto Chapí. Publicó en prensa varios trabajos sobre Clarín, luego reunidos en un tomo con el que inauguró la Biblioteca de Vulgarización Literaria que comenzó a dirigir en 1896. En los inicios de El Pueblo (Valencia), periódico que dirigía Blasco Ibáñez, se encargó de la parte literaria.
} 
grandes emociones a algo mayor que ellas, a lo verdaderamente inmenso, a lo que nos envuelve y protege con su magnitud.

Tal argumento debió de conmover al público, que llamó al autor a escena el día del estreno, junto con los actores ${ }^{5}$. La obra se mantuvo varios días en cartel, siendo cada vez más aplaudida, «por los detalles de observación y la poesía y el encanto que respira» (Nuevo Mundo, 26-1-1911). Los periódicos de la capital se hicieron eco del éxito de aquella representación en Valencia, muy esmerada según el Heraldo del Madrid (23-1-1911), que fue interrumpida en varias ocasiones por aplausos y aclamaciones a Emilia Pardo Bazán (El Liberal, 19-1-1911).

Ocho años después, todavía en vida de la escritora, se estrenó la primera adaptación a la escena de El saludo de las brujas, novela publicada en 1898. La realizó el actor Francisco Fuentes, con el que doña Emilia tenía antigua $\operatorname{amistad}^{6}, \mathrm{y}$ fue estrenada en el Teatro Isabel la Católica de Granada, por la compañía de Margarita Xirgu, el 6 de marzo de 1919, a beneficio del propio Francisco Fuentes, entonces primer actor de la compañía.

Fue un estreno esperado, pues la noticia se conoció con anterioridad, gracias a una indiscreción, sin duda premeditada, que doña Emilia deslizó en una entrevista concedida a Ramón Martínez de la Riva para Blanco y Negro (17-11-1918) .

Cuando en marzo del año siguiente tuvo lugar el estreno, la prensa se hizo eco del gran éxito (La Acción, 7-3-1919), debido no solo a la interpretación, en particular de Margarita Xirgu y de Paco Fuentes (Heraldo de Madrid, 7-31919), así como de Rivero (La Acción, ibidem), sino al «indudable acierto en la adaptación escénica de la famosa novela» (La Alhambra, 15-2-1919). Todavía un mes más tarde, La Alhambra (15-3-1919) se ocupaba de nuevo de la versión teatral de El saludo de las brujas, a su juicio muy acertada, lamentando que Fuentes planeara retocarla, aligerando el tercer y último acto.

\footnotetext{
5 Fueron especialmente aplaudidos «la señora Pla, la señorita Xifrá y los señores Colom y Muñoz» (Heraldo de Madrid, 23-1-1911).

6 El actor granadino Francisco Fuentes (1870-1934) fue quien estrenó Electra de Galdós. Emilia Pardo Bazán le trataba desde hacía años. En octubre de 1905, cuando Fuentes se encontraba en gira por América, le envió doña Emilia el manuscrito de Las raíces, con intención de que se estrenara en el Teatro Payret de La Habana (El Noroeste, 24-10-1905), lo que finalmente no tuvo lugar.

7 «Por la noche no podré asistir a la Comedia, porque espero a Paco Fuentes, que me va a leer la adaptación teatral de una de mis novelas... — ¿De qué novela, condesa? — le hemos preguntado. —De El saludo de las brujas. La ha puesto en escena en tres actos. Pero conste que no acepto la menor responsabilidad... No; no hablemos del teatro... No tengo el menor motivo de simpatía hacia él...».
} 
El argumento de la novela encierra una gran carga dramática, que culmina precisamente en el desenlace de la obra y de la relación entre Felipe, príncipe heredero de Dacia en el exilio, sin esperanzas de llegar a reinar al inicio de la trama, y Rosario, sobrina del pintor Viodal, que está dispuesta a abandonarle para facilitar su acceso al trono, cuando los partidarios de su dinastía llegan hasta la Costa Azul en su busca, y ponen como condición para su acceso al trono su matrimonio con una princesa real. La tensión dramática, que no decae a lo largo de la novela, late en las palabras, en las reacciones y hasta en los silencios - Rosario calla su embarazo para no obligar a Felipe - de unos personajes, cuya complejidad psicológica los hace creíbles y potencialmente teatrales.

No sabemos si Fuentes llegó a cumplir su propósito de modificar su texto inicial. La adaptación debió de ser acertada, porque en la siguiente temporada, 1919-1920, la llevó en su gira por provincias la compañía del Teatro Español de Madrid, que dirigía Jacinto Benavente ${ }^{8}$, y en la de 1920-1921 se esperaba su representación en Madrid (La Correspondencia de España, 30-8-1920), aunque no hemos podido confirmar que se llevara a cabo.

Apenas tres años después de la muerte de Emilia Pardo Bazán, la compañía de Narcisín ${ }^{9}$, el niño prodigio entonces triunfante, que pasados los años sería conocido como el gran actor Narciso Ibáñez Menta, incorporó a su repertorio y llevó en su gira por España ;Meu fillo! Era la adaptación escénica, en un acto y cinco cuadros, de un cuento de doña Emilia, realizada por el escritor argentino José Díaz Franco ${ }^{10}$, con música del maestro Bernardino Terés (1882-1969), muy vinculado al mundo de la zarzuela, la revista y las varietés. Por la prensa sabemos que se representó en el Teatro Principal de Pontevedra el 12 de enero de 1924 (Aparicio, 2001), que en Zaragoza se estrenó en el Teatro-Circo en septiembre de ese mismo año (El Imparcial, 28-9-1924), y en el teatro Apolo de Valencia el 5 de marzo de 1925 (La Reclam, 8-3-1925).

8 El Orzán anunciaba su próxima representación en La Coruña el 23-7-1920. Los primeros actores eran Ricardo Calvo y Francisco Fuentes y las primeras actrices Carmen Moragas, Carmen Seco y Josefina Roca.

9 Narcisín, entonces niño prodigio de la escena, fue conocido en su madurez como Narciso Ibáñez Menta (1912-2004), actor de teatro, cine y televisión, padre de Narciso Ibáñez Serrador. De familia teatral, era hijo del actor argentino Narciso Ibáñez y de la tiple guipuzcoana Consuelo Menta.

10 No tenemos datos sobre el adaptador. Aunque en algún periódico se le llamó «notable literato gallego» (El Imparcial, 28-9-1924), era argentino, como Narciso Ibáñez, director de la compañía. Es posible que fuera alguien de su entorno, sin especial relevancia en el mundo de la dramaturgia. 
Cuando se representó en Madrid, Narcisín llevaba dos años ausente de los teatros de la capital, donde de nuevo se le recibió con los brazos abiertos.

El texto teatral de Díaz Franco se basa en el cuento La Pepona, modificado en función del niño actor que protagonizaría la obra. Así, Díaz Franco convierte en un niño, Pacorro ( $\mathrm{La} \mathrm{Voz}, 28-10-1925)$, a la niña protagonista del relato y, por alguna razón, traslada la acción de Madrid a Galicia ${ }^{11}$. Por eso, cuando el 2 de septiembre de 1925 se estrenó ;Meu fillo! en el madrileño Teatro del Centro, El Imparcial (3-9-1925) la calificó de «zarzuela de costumbres gallegas», admitiendo, como casi toda la prensa madrileña, que desconocía de qué relato de doña Emilia procedía la pieza. Blanco y Negro (13-9-1925) fue la única publicación que mencionó La Pepona como fuente de la obrita teatral, porque en sus páginas se había publicado el cuento el 2 de febrero de 1919, con bonitas ilustraciones de Méndez Bringa.

En el periódico cordobés $\mathrm{La}$ Voz (28-10-1925), con motivo de su estreno en el Teatro Duque de Rivas, encontramos las noticias más extensas sobre lo que fue el texto dramático, muy fiel al relato de doña Emilia en el aspecto argumental:

La obra [...] es un cuadrito de poesía. Las andanzas de un pobre niño, robado de su casa muy chiquito todavía por unos mendigos; las escenas del chico con sus raptores, a quien (sic) cree tíos; la revelación que el ciego le hace de su origen y la vuelta al hogar paterno, están trazadas con sencillez. y delicadeza admirables. La emoción que doña Emilia pusiera en las páginas de su cuento se transmite íntegra al espectador, al través de la oportuna adaptación del señor Díaz Franco. La música de Terés sobre motivos gallegos es inspirada y se escuchó con gusto.

Este periódico no desvela que cuando Pacorro, pasados los años, logra encontrar la casa familiar, solo es reconocido como hijo y admitido en ella porque identifica a su muñeca, la Pepona de su infancia y del título del cuento. Esta es la escena que recoge la fotografía publicada en El Imparcial (3-9-1925), que hoy nos permite asomarnos a aquella representación, en la que los personajes aparecen ataviados con el traje regional gallego. Sin embargo, de los comentarios sobre la obra publicados en ese mismo número de

11 Hacía muchos años, Ceferino Palencia había pedido a doña Emilia una comedia ambientada en Galicia, sin que ella llegara a encontrar el argumento adecuado. Eran los momentos en que todavía se sentía atraída por las candilejas, que al mismo tiempo la asustaban (cfr. Heraldo de Madrid, 3-9-1899). 
El Imparcial se deduce que la adaptación no hizo honor al relato del que procedía:

No conocemos el cuento inspirador que, indudablemente, solo en su última parte -la que pinta el recelo de los campesinos, el odio entre dos familias y el momento de tierna emoción de la muñeca de los juegos infantilesha sido respetado con fidelidad. En el resto de la obra, las imposiciones de la esquematización teatral deben haber apartado al autor demasiado de la acción narrada por la ilustre escritora.

Las críticas del estreno fueron más elogiosas para la interpretación del niño prodigio que para la adaptación. Mientras El Sol (3-9-1925) afirmaba que «Don José Díaz Franco, autor del libro, y el maestro Terés, compositor, han trabajado menos de lo que para representar su arreglo trabaja Narcisín, poniendo en cada frase su además y recalcando su intención sin ahorrar esfuerzo», Melchor Fernández Almagro publicaba una reseña demoledora en La Época de ese mismo día:

Anoche nos creímos en el salón de fiestas de algún colegio, llamado quizá de los Santos Inocentes. Lo menos infantil que había en el Teatro del Centro era precisamente Narcisín. ¡Con qué puerilidad se conmovían las gentes ante el cándido arreglo de un cuento de la condesa de Pardo Bazán! El señor Díaz Franco ha realizado su labor de adaptación, pensando seguramente que Meu fillo iba a ser puesto por los educandos del colegio a que antes aludimos. Como Narcisín es el muchacho más aventajado, asumió el papel de protagonista, secundado por unos cuantos compañeros de clase y por algún que otro hermano repetidor.

Seguramente que luego - nosotros no pudimos esperar al final del acto-fueron repartidos los premios y que se obtuvo una fotografía. Narcisín, a la derecha del padre Superior, lucía una vistosa banderola. M. F. A.

Es posible que, después de este varapalo recibido en Madrid, la compañía retirara ;Meu fillo! del repertorio, pues no hay noticias posteriores de su representación.

En la temporada 1925-1926, la compañía de José Romea estrenó en el Gran Teatro de Córdoba ${ }^{12}$ una nueva adaptación de El saludo de las brujas, ahora en cuatro actos, realizada por dos periodistas vallisoletanos, Federico

12 Es posible que se hubiera representado con anterioridad en otras localidades, pero esta es la noticia más antigua que he encontrado del estreno de esta obra. 
Santander ${ }^{13}$ y José María Vela de la Huerta ${ }^{14}$. La reacción de un sector de la prensa cordobesa fue adversa, sobre todo por razones morales. Un suelto en El Defensor de Córdoba (22-2-1926) firmado por Morsamor en la sección de teatros lamentaba que «unos arregladores de dramas» hubieran escenificado la novela de doña Emilia con tan poco arte que los finales del segundo y tercer acto eran muy parecidos. Pero la mayor objeción residía en la inmoralidad del argumento, tan impropio de la autora de San Francisco de Asís:

La vida de... idilio - le llaman así por eufemismo los arregladores-del príncipe y Rosario merece una acerba censura, porque de ahí a presentar como legítimo el amor libre no hay más que un paso.

El 31 de marzo, la Revista Mariana de la misma ciudad incluía El saludo de las brujas en una larga relación de «Obras que constituyen un serio peligro para la moral».

No obstante, en la siguiente temporada, la compañía de Carmen Seco puso en escena El saludo de las brujas en Valladolid, después de haberla llevado por varias capitales de España y América (El Norte de Castilla, 3-11-1926). Del estreno en el Teatro Calderón de Valladolid, el 3 de noviembre de 1926, y de la buena acogida del público, hablaba José María de Cossío a Gerardo Diego en una carta fechada en La Casona de Tudanca el día 11: «Federico Santander y Pepe Vela estrenaron en Valladolid, con éxito favorable, un arreglo de El saludo de las brujas, de la Pardo Bazán» (Diego 1996: 143). El éxito en esta ocasión no se debió tanto a los actores, que «realizaron una labor discreta y acoplada» (El Norte de Castilla, 4-11-1926), como al interés de la trama y al acierto de la adaptación. El papel de Rosario lo interpretó Carmen Seco, Pablo Álvarez Rubio fue el príncipe Felipe, y Francisco Ros el bohemio Yalomitsa.

Santander y Vela manifestaron su deseo de honrar a Emilia Pardo Bazán en un artículo titulado «Homenaje», que el mismo día del estreno publicaron en la primera plana de El Norte de Castilla, acompañado de un retrato

13 Federico Santander, periodista vallisoletano nacido en 1883, declaradamente monárquico, fue redactor de El Norte de Castilla y posteriormente de $A B C$, y colaborador de otros periódicos de provincias. Su muerte tuvo lugar en los primeros momentos de la guerra civil. Con José M. ${ }^{a}$ Vela escribió varias obras de teatro, como La casa de Luján (1928), ;Por el nombre...! (1928) o esta adaptación de El saludo de las brujas (1924).

14 José María Vela de la Huerta, periodista, escritor y crítico teatral vallisoletano, es autor de $D e$ telón adentro. Memorias de un exfarandulero (1917). Se pierde su rastro biográfico a comienzos de la guerra civil. 
de la escritora. A ella le dedicaban de antemano los posibles aplausos que pudieran sonar en el Teatro Calderón. De hecho, en la representación, al final de cada acto se proyectó en la pantalla el retrato de doña Emilia, al que efectivamente el público aplaudió con entusiasmo (Cfr. El Norte de Castilla, 4-11-1926).

Francisco de Cossío, refiriéndose a las dificultades que suelen ofrecer las adaptaciones, destacaba en una extensa reseña en El Norte de Castilla (4-11-1926) que:

no es en las narraciones de la condesa de Pardo Bazán donde estas dificultades se hacen insuperables. En todos los cuentos y novelas de doña Emilia existe una línea de acción fundamentalmente dramática y esta acción se desenvuelve en un diálogo fluido, natural y humano. Muchas de las narraciones de la ilustre escritora parecen pensadas para la escena [...] El saludo de las brujas posee esta línea de acción dramática quizá más acentuada que ninguna otra novela de doña Emilia [...] Toda esta lucha, profundamente dramática, halla en el proscenio un marco más fuerte y vigoroso que en el libro.

Esta reseña nos acerca a lo que fue aquella obra teatral, que no llegó a imprimirse, y que, en cuanto al contenido, parece seguir fielmente la novela, «sobria en descripciones, prolija en diálogos, tipos de muy buena caracterización, y efectos brillantes que aprovechar para el final de los actos». Según Cossío, «el éxito culminó al final del segundo acto, el de una teatralidad más evidente». Pero no llega a explicar cómo se resuelve la escena final, en la que hubo que «forzar un poco la verdad convencional, para traer al protagonista a representar la escena postrera de su vida», y que, según el crítico, aportó a la producción escénica «una solución melodramática, imprescindible, sin embargo, pues en ella, quizá, se encierra la intención del título y su esencia simbólica» ${ }^{15}$.

La compañía de Carmen Seco y Francisco Ros llevó El saludo de las brujas de Santander y Vela a otras ciudades castellanas. En diciembre la representaron en el Teatro Alfageme de León (El Norte de Castilla, 26-12-1926), donde El Diario de León destacaba que, sobre todo el tercer acto, era un modelo de técnica teatral, y que los aplausos del público habían hecho levantar el

15 Como es sabido, el título de la novela de Emilia Pardo Bazán hace referencia a las palabras que pronuncia una de las brujas, en la escena III del acto primero de la tragedia de Shakespeare, dirigiéndose a Macbeth: «Tú serás rey». 
telón hasta cuatro veces. A mediados de febrero, la misma compañía la estrenaba en el Coliseo Castilla de Burgos (El Norte de Castilla, 17-2-1927).

Bastantes años separan esta segunda versión dramática de El saludo de las brujas del siguiente estreno de una adaptación escénica de una obra narrativa de Emilia Pardo Bazán. En este caso mejor sería hablar de una obra teatral inspirada en un cuento de la escritora, por la libertad con que actuó Francisco Serrano Anguita ${ }^{16}$ al componer su comedia Todo Madrid. La noticia saltó a la prensa por una entrevista que Blanca Quiroga, la hija mayor de la escritora, concedió a Plaudere ${ }^{17}$. Afirmaba la marquesa de Cavalcanti: «Serrano Anguita ha escrito una comedia cuyo primer acto tiene por asunto el mismo del cuento de mi madre El décimo. Aunque va a hacerlo constar así, me ha pedido autorización».

Es esta la única de las adaptaciones que fue publicada y, por tanto, la única que podemos juzgar en el aspecto textual, en el que se invierten las habituales características del texto dramático, de ordinario más sintético, frente a las del narrativo, que suele ser más extenso.

Aquí ocurre lo contrario. Los tres personajes del relato - ninguno tiene nombre propio- se convierten nada menos que en catorce en la comedia. El cuento, narrado en primera persona y comenzando por el final, era poco más que una anécdota. Entre el comienzo — «iLa historia de mi boda? Óiganla ustedes; no deja de ser rara»—y el final — «Lo más notable de esta historia es que he sido feliz»—, el protagonista relata cómo llegó a casarse con una chiquilla que, a altas horas de una noche madrileña, le había vendido un décimo de lotería que resultó premiado.

En la comedia, el protagonista no solo tiene nombre, Jacinto Carvajal, sino padre, don Felipe, amigos y criados que lo atienden en Madrid, pues los Carvajal de la obra teatral son de origen andaluz y pertenecen a la buena sociedad, a ese Todo Madrid del título de la comedia. También la vendedora de lotería tiene nombre, Trini, y una hermana que la acompaña, Mercedes, además de amigos y conocidos.

16 Francisco Serrano Anguita (Sevilla, 1887- Madrid, 1968) fue periodista y autor de unas cincuenta obras teatrales, algunas de ellas escritas en colaboración. La más conocida hoy es probablemente la zarzuela Black el payaso, con partitura de Pablo Sorozábal.

17 Plauderer era uno de los seudónimos de Manuel Jiménez Moya (1877-1947), periodista guipuzcoano y autor dramático, fundador, director y redactor de diversos periódicos de ámbito nacional, y director durante muchos años de la revista Lecturas, donde se publicó esta entrevista (Primer semestre de 1944). 
La localización de los acontecimientos está poco más que abocetada en el cuento, mientras que en la comedia los tres actos se desarrollan respectivamente en el piso de Jacinto, en el restaurante La Quinta de Goya, a orillas del Manzanares, y nuevamente en el domicilio del protagonista. Además, el adaptador aportó a la obra comicidad, costumbrismo y hasta gracejo en los diálogos, que no abundan en el cuento.

No obstante, lo más destacable de la adaptación es el giro de ciento ochenta grados que Serrano Anguita imprimió al argumento inicial. El escritor supo ver las posibilidades dramáticas del cuento, y resolvió, sin embargo, en sentido inverso el conflicto planteado.

Si el protagonista del cuento relataba en él la historia de su boda, es precisamente la boda lo que falta en la comedia, pues la lotera Trini, consciente de su posición en la vida, no acepta casarse con el señorito Jacinto. En este sentido el desenlace del conflicto resulta más realista, más cercano a la vida real, en la comedia que en el cuento. Serrano Anguita, sin embargo, evita cualquier sombra de clasismo poniendo en boca de don Felipe las palabras con que concluye la obra, dirigidas a su hijo:

Tú aguántate, que no te la mereses. Eres tú poco premio para la que nos hizo tan buena obra. Cuando quieras navegar... te embarcas en el estanque del Retiro. Aqui te ha echado por la borda esta mosita del mantonsiyo y el pañuelo blanco... que está muy alta para ti. ¿Qué vamos a haserle, niño? ¡Pasiensia!

La comedia fue estrenada el 29 de septiembre de 1944, en el Teatro de la Zarzuela de Madrid $^{18}$, por la compañía de Antonio Vico, que dio vida al protagonista. Le acompañaban en escena Carmen Carbonell, la joven Paquita Gallego y Manuel González, que era además el director de escena. Los demás intérpretes fueron Julio Sanjuán, María Luisa Arias, Matilde Galiana, Carmen Villa, Mercedes Sillero, Manolita Henche y los actores Puga, Alburquerque, Blanch y Santonja. Fueron muy celebrados los decorados de Redondela (ABC, 30-9-1944).

En cuanto a la recepción, me remito en este caso a la crónica entusiasta de Alfredo Marquerie (ibidem), en la que destacaba entre otras cosas que:

18 El 17 de agosto se había estrenado en el Teatro Victoria Eugenia de San Sebastián (cfr. Barcelona Teatral, 24-8-1944) y es posible que también se hubiera representado en otras localidades. 
el público, después de subrayar con rumores admirativos la genial —así, como suena - creación que hace en el papel a él encomendado el gran actor Antonio Vico, le tributó una de las más grandes ovaciones que hemos escuchado en un estreno teatral.

La última de las adaptaciones de una obra narrativa de Emilia Pardo Bazán al teatro se llevó a cabo en 1951, con ocasión del centenario de su nacimiento. La hizo Gerardo Gasset Neyra ${ }^{19}$, convirtiendo en obra dramática titulada El solterón la novela de 1896 Memorias de un solterón. Hasta donde sabemos, solo se puso en escena, y en una sola ocasión, el primer cuadro del segundo acto, como ilustración de la conferencia que el 16 de mayo pronunció el autor en el Centro Gallego de Madrid, sobre «La Pardo Bazán y el teatro», inaugurando con ella un ciclo en homenaje a la escritora ${ }^{20}$.

En su conferencia, cuyo original mecanografiado se conserva en el archivo de la Real Academia Galega, el autor explica que eligió precisamente ese cuadro por dos razones. La primera, «porque es donde las exigencias teatrales me han parecido respetar más miméticamente el espíritu y la letra de su gloriosa autora». La segunda razón era por lo mucho que en ese cuadro encontraba él de la joven doña Emilia, cuando esta intentaba afirmar su personalidad y sus ideales en medio de un ambiente poco proclive a admitir la valía de una mujer.

Para la mejor comprensión del cuadro, Gasset Neyra ponía en antecedentes a los espectadores, explicándoles que el primer acto - que no verían- se había desarrollado en el despacho de don Benicio Neira ${ }^{21}$. Allí, tres de sus numerosas hijas, Rosa, Argos y Feíta, estaban preparando una soirée, cuando entra don Benicio, acompañado de Mauro Pareja, el solterón y confidente de los muchos problemas económicos del sufrido padre de fa-

19 Gerardo Gasset Neyra, nieto de Eduardo Gasset y Artime y primo de José Ortega y Gasset, nació en A Pobra do Caramiñal en 1899. Fue abogado y diplomático, carrera de la que se apartó en 1939. Escribió en diversos periódicos, entre ellos El Imparcial, vinculado a su familia. En su juventud trató a Valle-Inclán y le dedicó una de sus primeras novelas, A la deriva (1925), a la que había precedido Impresionable (1923). Como autor teatral escribió, entre otras obras, Nuestra Señora de Gundián (1945) y El síncope de Dorotea (1953), basada en La Dorotea de Lope de Vega.

20 Las demás conferencias del ciclo las impartieron Victoriano García Martí («La Pardo Bazán dentro del cuadro de las mujeres gallegas»), Dionisio Gamallo Fierros («La Pardo Bazán y la poesía. Sus versos y su crítica de poetas»), Camilo José Cela («Doña Emilia como novelista»), el padre Legísima (sin título), Elena Quiroga («La Pardo Bazán y los niños») y Wenceslao Fernández Flórez, que clausuró el ciclo de conferencias en el Centro Gallego (cf. ABC, 16-5-1951 y Hoja del Lunes, 21-51951).

21 Este personaje apareció por vez primera en la novela Doña Milagros, donde se contaban con detalle los antecedentes de su situación actual. 
milia. Este le cuenta la compleja y comprometida situación en que se encuentra, y cuál es la única salida que ve a los problemas que le acucian. El acto lo cerraba Feíta, la intelectual e independiente, en una escena de rebeldía ante las mentiras y falsedades del mundo que la rodeaba, ella que se sentía capaz de todo por defender la verdad.

El escenario es otro en el segundo acto. Cuando comienza el cuadro que ilustraría la conferencia, «Mauro Pareja, el solterón, se ha replegado en sus cuarteles a su soledad y a los cuidados regalones de D. ${ }^{a}$ Consola», personaje tras el cual adivina Gasset Neyra a la antigua doncella de doña Juana de Vega, la viuda de Espoz y Mina. A partir de esta información, no cabe más que imaginar el resto de la obra si, como parece, siguió de cerca el texto de la novela. La prensa a la que hemos tenido acceso no recogió ningún eco de aquella representación. El espacio escénico debió de ser bastante reducido, pues Gasset Neyra pidió disculpas a los espectadores, confiando en que con su imaginación pudieran suplir las deficiencias de la puesta en escena. Quienes pusieron todo de su parte fueron la joven Ángela Pla, que interpretó el papel de Feíta, Ángel Terrón, Juana Azorín, Julián Fuentes, Carlos Luis Ponte y Molinero.

Hasta aquí, las noticias que hemos podido recabar sobre las adaptaciones de obras narrativas de Emilia Pardo Bazán a la escena, en un arco temporal de cuarenta años. Los juicios proceden en muchos casos de la prensa del momento y no siempre son objetivos. Pero esas informaciones son las que nos permiten hoy conocer un nuevo aspecto de la proyección que tuvo la creación literaria de doña Emilia. La novelista volvió a subir a los escenarios cuando ya sus tentativas dramáticas eran agua pasada — «El teatro me ha dejado un desapacible recuerdo» (La Nación, 10-12-1916)—, aupada por quienes admiraron su creación literaria, aunque no estuvieran a la altura de su talento. De hecho, casi todas estas adaptaciones implican el deseo de rendir homenaje a una novelista cuya escritura encierra grandes posibilidades dramáticas, a pesar de no haber triunfado cuando quiso hablar a sus contemporáneos desde la altura de los escenarios.

\section{REFERENCIAS BIBLIOGRÁFICAS}

APARICIO MORENO, Paulino (2001). La vida escénica en Pontevedra: 1901-1924. Madrid: UNED (en microforma. El texto completo puede leerse en la web del Centro de Investigación de Semiótica Literaria, Teatral y Nuevas Tecnologías: http://www.uned.es/centro-investigacionSELITEN@T/pdf/AparicioMorenoPaulino.pdf. 
DE VAL, Mariano Miguel (1906). «Los novelistas en el teatro». Ateneo 1, 62-65.

DIEGO, Gerardo (1996). Epistolario: nuevas claves de la generación del 27. Alcalá de Henares: Universidad de Alcalá de Henares-Madrid: Fondo de Cultura Económica.

FREIRE, Ana María (2013). «Emilia Pardo Bazán y el género periodístico de la entrevista». Studi Ispanici XXXVIII, 197-224.

NIEVA, Francisco (1989). «Una mirada sobre el teatro de Emilia Pardo Bazán». En Estudios sobre «Los pazos de Ulloa», Marina Mayoral (ed.), 189-201. Madrid: Cátedra-Ministerio de Cultura.

PARDO BAZÁN, Emilia (1906). «Los novelistas en el teatro». Ateneo 2, 181-184.

- (2010). Teatro completo (Estudio preliminar, edición y notas de Montserrat Ribao Pereira). Madrid: Akal.

SERRANO ANGUITA, Francisco (1944). Todo Madrid. Madrid: Gráficas Cinema.

Recibido el 1 de junio de 2013.

Aceptado el 19 de septiembre de 2013. 
